\title{
Nonlinear multivariable analysis of SOI and local precipitation and temperature
}

\author{
Y.-H. Jin ${ }^{1}$, A. Kawamura ${ }^{2}$, K. Jinno ${ }^{2}$, and R. Berndtsson ${ }^{1}$ \\ ${ }^{1}$ Department of Water Resources Engineering, Lund University, Box 11822100 Lund, Sweden \\ ${ }^{2}$ Institute of Environmental Systems, Kyushu University, Fukuoka, Japan \\ Received: 18 June 2004 - Revised: 6 September 2004 - Accepted: 9 September 2004 - Published: 21 January 2005 \\ Part of Special Issue "Nonlinear deterministic dynamics in hydrologic systems: present ativities and future challenges"
}

\begin{abstract}
Global climate variability affects important local hydro-meteorological variables like precipitation and temperature. The Southern Oscillation (SO) is an easily quantifiable major driving force that gives impact on regional and local climate. The relationships between SO and local climate variation are, however, characterized by strongly nonlinear processes. Due to this, teleconnections between globalscale hydro-meteorological variables and local climate are not well understood. In this paper, we suggest to study these processes in terms of nonlinear dynamics. Consequently, the nonlinear dynamic relationship between the Southern Oscillation Index (SOI), precipitation, and temperature in Fukuoka, Japan, is investigated using a nonlinear multivariable approach. This approach is based on the joint variation of these variables in the phase space. The joint phasespace variation of SOI, precipitation, and temperature is studied with the primary objective to obtain a better understanding of the dynamical evolution of local hydro-meteorological variables affected by global atmospheric-oceanic phenomena. The results from the analyses display rather clear loworder phase space trajectories when treating the time series individually. However, when plotting phase space trajectories for several time series jointly, complicated higher-order nonlinear relationships emerge between the variables. Consequently, simple data-driven prediction techniques utilizing phase-space characteristics of individual time series may prove successful. On the other hand, since either the time series are too short and/or the phase-space properties are too complex when analysing several variables jointly, it may be difficult to use multivariable statistical prediction techniques for the present investigated variables. In any case, it is essential to further pursue studies regarding links between the SOI and observed local climatic and other geophysical variables even if these links are not fully understood in physical terms.
\end{abstract}

Correspondence to: Y.-H. Jin

(jin.younghoon@tvrl.lth.se)

\section{Introduction}

Global climatic variation and warming are expected to result in significant changes in local and regional climate. It is especially local temperature and precipitation patterns that are expected to significantly deviate from the present-day levels in case of a significant future global warming. The Southern Oscillation Index (SOI) is an easily quantifiable climatic parameter that can be used to measure the strength of the atmospheric signal in local and regional climatic data. The SOI is defined as the normalized difference in surface pressure between Papeete at Tahiti in central Pacific Ocean and Darwin in northern Australia. The SOI characterizes the inter-annual atmospheric seesaw phenomenon called Southern Oscillation (SO). Quantitative links between the SOI and observed local climatic and other geophysical variables are important to establish because they can be used as early indicators of near-term extreme weather or long-term effects on available water resources (e.g. Chiew et al., 1998).

The El-Niño - Southern Oscillation (ENSO) phenomenon has been shown to affect large-scale temperature and weather patterns for many areas of the world (e.g. Gordon, 1986; Opoku-Ankomah and Cordery, 1993; Uvo et al., 1998). Similarly, during recent years strong connections between ENSO and large-scale hydrological and hydro-meteorological anomalies have been demonstrated (e.g. McBride and Nicholls, 1983; Moss et al., 1994; Uvo and Graham, 1998). Hense (1987) hypothesized the existence of a chaotic strange attractor for several ENSO related monthly time series. Similarly, several ENSO models have been shown to exhibit chaotic properties (Jin et al., 1994; Tziperman et al., 1994). Elsner and Tsonis (1992) analyzed time series of the El-Niño - Southern Oscillation and found a significant nonlinear structure on the monthly time scale. Kawamura et al. (1998) on the other hand, found no evidence of chaos when analyzing monthly Southern Oscillation Index for 130 years. 

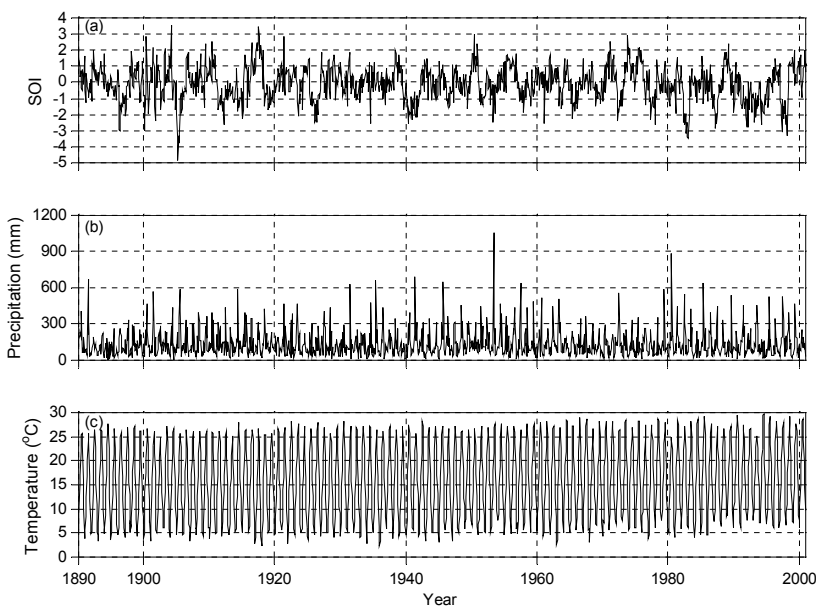

Fig. 1. Time series plots of raw monthly (a) SOI, (b) precipitation, and (c) temperature.

As indicated from the above, finding quantitative links between the SOI and local climatic variables may be expected to be hampered by the strong nonlinearities involved when studying relationships between joint atmosphere-ocean-land surface data. Recently, Kawamura et al. (2000) showed that categorized extreme SOI displays corresponding elevated values of temperature and precipitation in south Japan. Consequently, it has been shown that certain kinds of co-variation exist between SOI and local temperature and precipitation. This type of relationship, however, is not easily distinguished using simple linear statistics. Consequently, there are needs to develop methods that can display complicated nonlinear relationships among several variables simultaneously. We thus, attempt to further investigate these relationships for SOI and local temperature and precipitation in south Japan.

In this paper we investigate nonlinear relationships between SOI, temperature, and precipitation at Fukuka, Japan, using a multivariable dynamical approach. The method, which is based on chaos theory, has been significantly developed during the past two decades (see e.g. Sivakumar, 2004). Our main interest is focused towards simultaneous temporal variation in the span 10-100 years. The approach involves investigation of the dynamical variational pattern in phase space after standardization and nonlinear noise reduction. In the first parts of the paper we outline the data transformation and methodology. Then follow analyses using joint phase space trajectories and correlation dimension estimation. We close with a summary and discussion of practical implications.

\section{Data used}

Monthly time series of SOI, precipitation, and temperature are used to investigate joint phase space relationships. The SOI data are calculated using the mean sea level pressure (MSLP) at Papeete, Tahiti $\left(149.6^{\circ} \mathrm{W}, 17.5^{\circ} \mathrm{S}\right)$ in central Pacific Ocean and Darwin $\left(130.9^{\circ} \mathrm{E}, 12.4^{\circ} \mathrm{S}\right)$ in northern
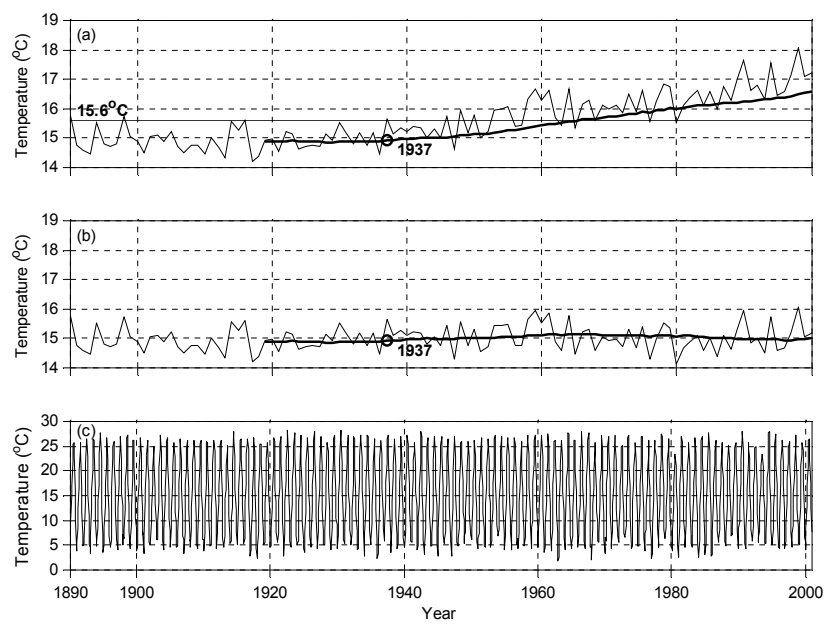

Fig. 2. Trend-removal for temperature data from 1937: (a) annual mean temperature and its thirty-year moving average, (b) annual mean temperature and its thirty-year moving average after linear trend was removed, and (c) trend-removed monthly temperature.

Australia. The MSLP data starting from 1882 are available through web sites such as NOAA/National Weather Service (www.cpc.ncep.noaa.gov/data/indices). However, in the present study, we use MSLP data from 1866 augmented by Ropelewski and Jones (1987), and Allan et al. (1991). Two commonly used methods to compute SOI from MSLP at Tahiti and Darwin are Troup's method (Troup, 1965; McBride and Nicholls, 1983) and the Climate Prediction Centre's method (Ropelewski and Jones, 1987). The difference between the two methods is very small as pointed out by McBride and Nicholls (1983), Ropelewski and Jones (1987), and Kawamura et al. (1998, 2001). Therefore, in the present study, only the Troup's SOI time series are used. Troup's method first takes the difference between pressures at Tahiti and Darwin. Then the difference series is normalized to mean zero and standard deviation one by subtracting the monthly mean values and dividing with the monthly standard deviations using a base period (usually 1951-1980) for the computation of the mean and standard deviation. This normalized time series is defined as Troup's SOI and used in the present study (Fig. 1a).

Monthly precipitation and temperature data at Fukuoka, Japan, are selected because of long and well-established observations. The annual mean for precipitation here is $1627 \mathrm{~mm}$, while the annual mean temperature is $15.6^{\circ} \mathrm{C}$. The time series plots are shown in Figs. $1 \mathrm{~b}$ and 1c. The monthly temperature data, however, display clear positive linear trend from about 1937. Therefore, mean annual values and thirtyyear moving averages are calculated to remove the trend (see Fig. 2). It is likely that this linear trend is an effect of urbanization. For all variables, the data periods are from 1890 to 2000 . 

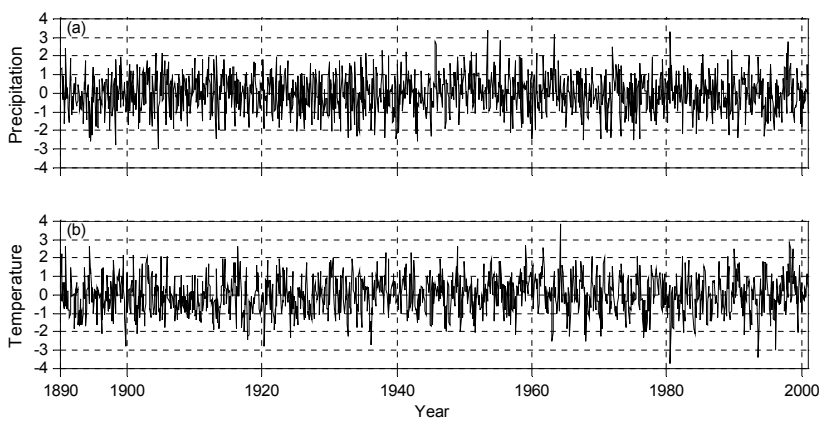

Fig. 3. Normally standardized data for (a) precipitation and (b) temperature.

\section{Data transformation}

In order to be able to compare the present nonlinear analyses results with previous analyses involving linear statistical techniques (see Kawamura et al., 2000), we perform similar data transformation below for the same data. The SOI data are used directly without transformation due to its already normal distribution. However, the precipitation and temperature data display positive skewness and annual periodicity (e.g. Jin et al., 2002a, b). Therefore, precipitation, and temperature data are normalized and standardized to remove deterministic components, i.e. seasonality and annual periodicity (Salas, 1993). The monthly precipitation data are normalized by a cubic root transformation. The normalized precipitation is then standardized to zero mean and standard deviation of one (Fig. 3a). The temperature data are similarly standardized to zero mean and standard deviation of one (Fig. 3b; e.g. Jin, 2004). The standardization with normalization of the local variables is not expected to alter the dynamical co-evolution of deterministic components in the time frame for the different variables. In other words, the variations around the mean including extreme values or outliers in the data are mainly considered in the present study.

The next procedure is to calculate accumulated deviations from mean for all time series. This is done in order to display general quasi-periodical characteristics in time domain. The resulting time series are shown in Figs. 4a-4c. Generally, observed geophysical time series data contain a substantial amount of noise. Therefore, the data need to be cleaned by a noise reduction scheme (e.g. Grassberger et al., 1991). Moving average and low-pass filter are commonly used methods for noise reduction. In the present study, however, we use a nonlinear noise reduction scheme specifically developed for deterministic dynamics studies proposed by Schreiber (1993). The general idea of the nonlinear smoothing is to replace each coordinate in the time series $\left\{x_{i}\right\}, i=1, \ldots, T$, by an average value over a suitable neighborhood in the phase space. The neighborhoods are defined in a phase space reconstructed by delay coordinates. To define the neighborhoods, first fix the positive integers $k$ and $l$, and construct
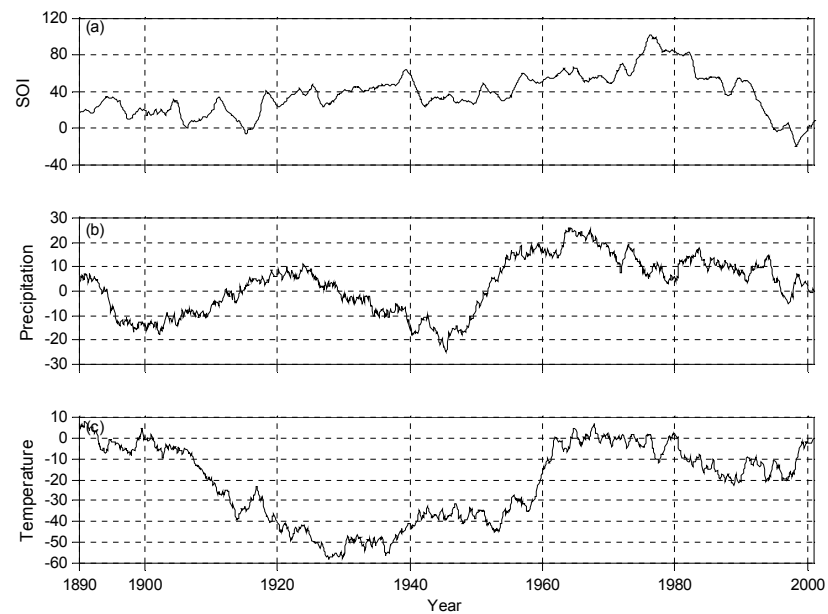

Fig. 4. Accumulated deviations from mean for (a) SOI, (b) precipitation, and (c) temperature.

embedding vectors $\boldsymbol{X}_{i}$.

$\boldsymbol{X}_{i}=\left[x_{i-k}, \ldots, x_{i+l}\right]$.

A radius $\eta$ is chosen for the neighborhoods. For each coordinate in $X_{i}$ find the set $\Omega_{i}^{\eta}$ of all neighbors $X_{j}$ for which

$\sup \left\{\left|x_{j-k}-x_{i-k}\right|, \ldots,\left|x_{j+l}-x_{i+l}\right|\right\} \equiv\left\|X_{j}-X_{i}\right\|_{\text {sup }}<\eta$,

where the symbol "sup" denotes the highest value of the elements. Consequently, the present coordinate $x_{i}$ is replaced by its mean value in $\Omega_{i}^{\eta}$ :

$x_{i}^{\text {coor }}=\frac{1}{\left|\Omega_{i}^{\eta}\right|} \sum_{\Omega_{i}^{\eta}} x_{j}$.

Here, $k$ and $l$ are both selected to 12 months. As a result, the first and last 12 months of the monthly time series are not noise-reduced, and the unchanged periods are neglected in the following analysis. The resulting noise-reduced monthly time series are shown in Figs. 5a-5c.

\section{Phase space analysis}

Dissipative dynamical systems exhibiting chaotic behavior generally display strange attractors in the phase space (Grassberger and Procaccia, 1983). The attractor can be examined in the phase space by using the method of time-delay coordinates. For this, time series are plotted versus the same series but with a time delay on the other axes. In mathematical terms, let $\left\{x_{i}\right\}$ be a discrete sample time series. A state space vector $\boldsymbol{X}_{i}$ is constructed, or embedded from $m$ consecutive values of the time series into a phase space whose coordinates are described by

$\boldsymbol{X}_{i}=\left[x_{i}, x_{i+\tau}, \ldots, x_{i+(m-1) \tau}\right]$

where $\tau$ is the delay time, and the dimension $m$ of the vector is known as the embedding dimension. Following Eq. (4), a 

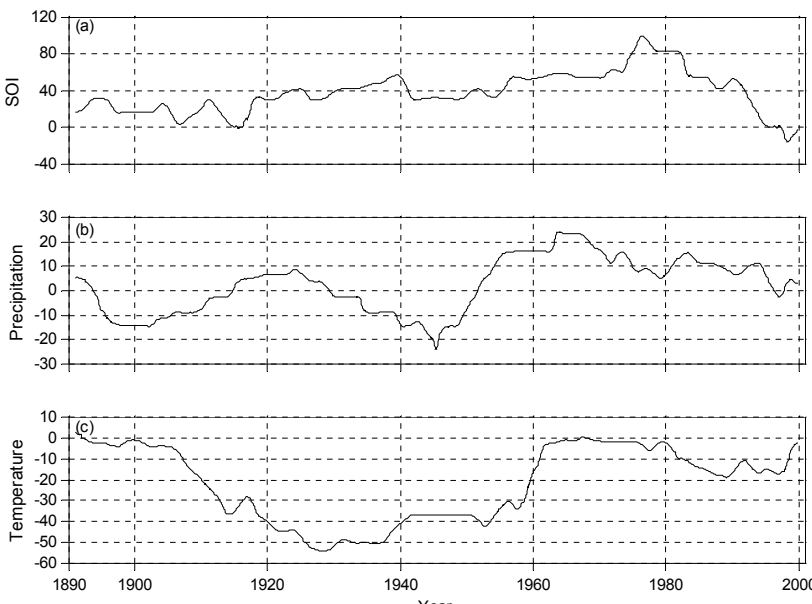

Fig. 5. Noise-reduced monthly time series of the accumulated deviations from mean for (a) SOI, (b) precipitation, and (c) temperature.

new time series of the state space vector $\boldsymbol{X}_{1}, \boldsymbol{X}_{2}, \ldots, \boldsymbol{X}_{N}$ is generated. Each vector $\boldsymbol{X}_{i}$ describes a point in an $m$ dimensional phase space. Thus, the sequence of these vectors defines a trajectory in time.

Figures $6 \mathrm{a}-6 \mathrm{c}$ show phase space trajectories for SOI, precipitation, and temperature, respectively, as previously defined. In the figures an embedding dimension equal to three (i.e. $m=3$ ) was used. From the figures it can be seen that this embedding dimension efficiently visualizes the dynamical evolution of the data used for the study. The generated time series for the state space vectors are connected with straight lines to indicate the continuous time evolution, even though the transition values between one space vector and the next are not known. For all variables, the delay time $\tau$ was selected to four months. As seen from the figures all time series display a relatively clear evolution for this delay time in the three-dimensional phase space.

If a time series contain chaotic properties, the state vector $\boldsymbol{X}_{i}$ will be attracted to a particular region in the phase space known as the strange attractor (see e.g. Jinno et al., 1995; Berndtsson et al., 1994). The attractor may, however, be completely concealed if the time series contain noise. Therefore, it is important to clean the time series before any type of analysis.

The phase space plots of SOI, precipitation, and temperature as seen in Fig. 6 appear to display regions of recurring visits in time domain. These may indicate the region which could hold a strange attractor. Due to the rather short time series, however, the number of recurring visits in phase is also rather small and it is therefore difficult to make an exhaustive analysis.

Corresponding plots for joint phase space plots are shown in Fig. 7. As seen from the figure the phase space trajectories for these relationships are much more complicated and do not display any obvious relationships. Consequently, if relationships exist, they contain higher-order nonlinearities. The diagrams can, however, be used to show similarities be-
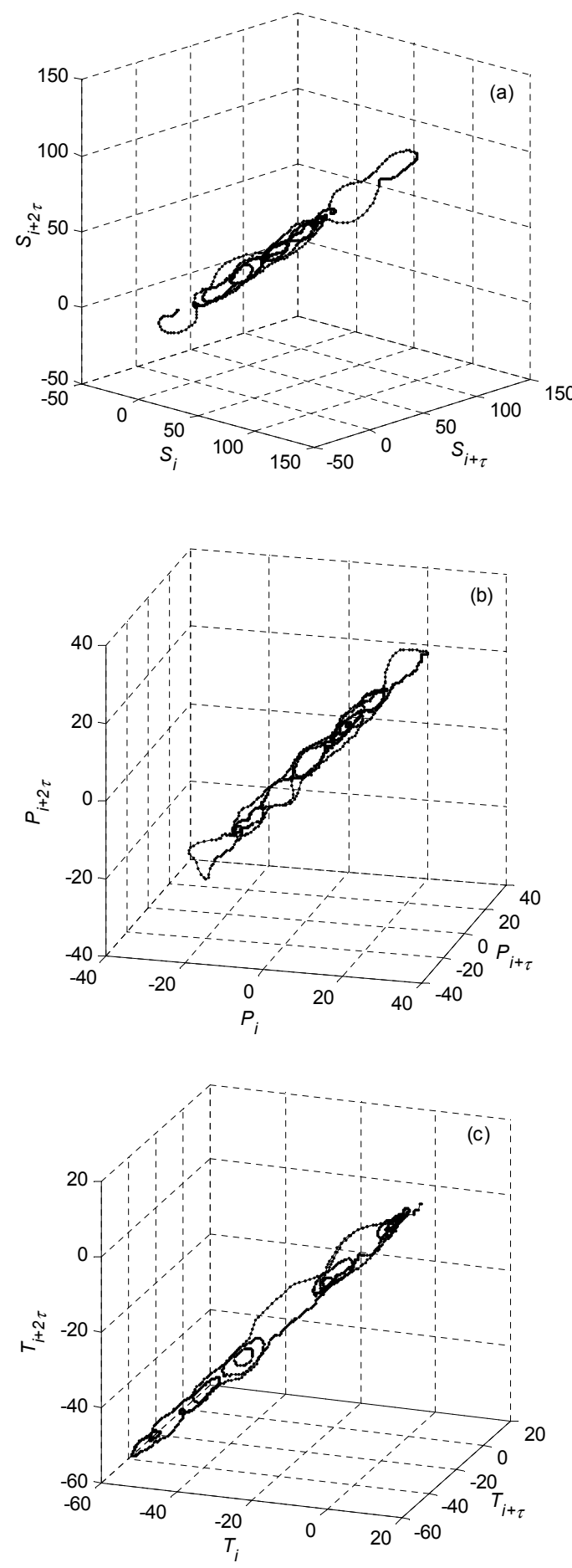

Fig. 6. Phase space plots for (a) SOI, (b) precipitation, and (c) temperature with delay time $\tau=$ four months, respectively.

tween different co-evolving climatic variables and driving climatic indicator such as the SOI. For precipitation and temperature it can be seen that years that represent a local minimum and/or maximum in the cumulative time series diagrams (Fig. 5) is clearly displayed in the joint phase space diagrams. Examples of such situations are the years 1945, 1963, 1976, and 1998 for precipitation. Corresponding years 

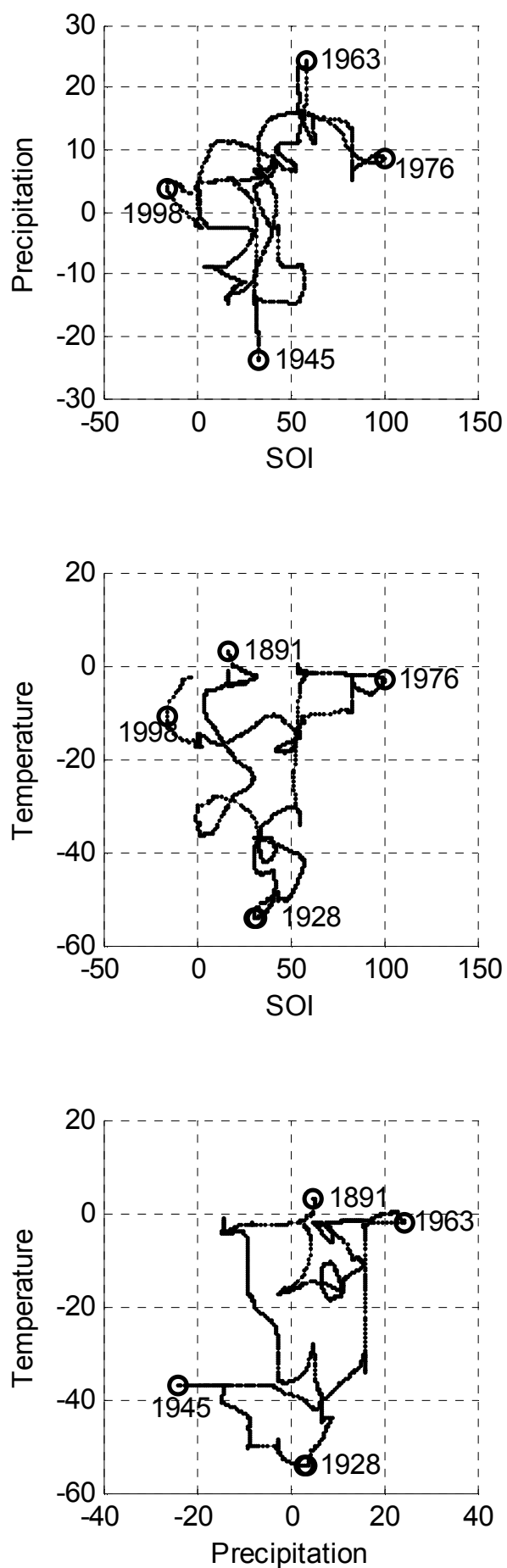

Fig. 7. Joint phase space plots of SOI, precipitation, and temperature $(\tau=0)$.

for temperature are 1891, 1928, 1976, and 1998. For these years SOI displayed maximum for 1976 and minimum for 1998. For the years 1891, 1928, 1945, and 1963, SOI displayed intermediate values. When comparing joint precipitation and temperature phase space trajectories, 1891 and 1963 were years when both variables displayed large maxima and 1928 and 1945 large minima.

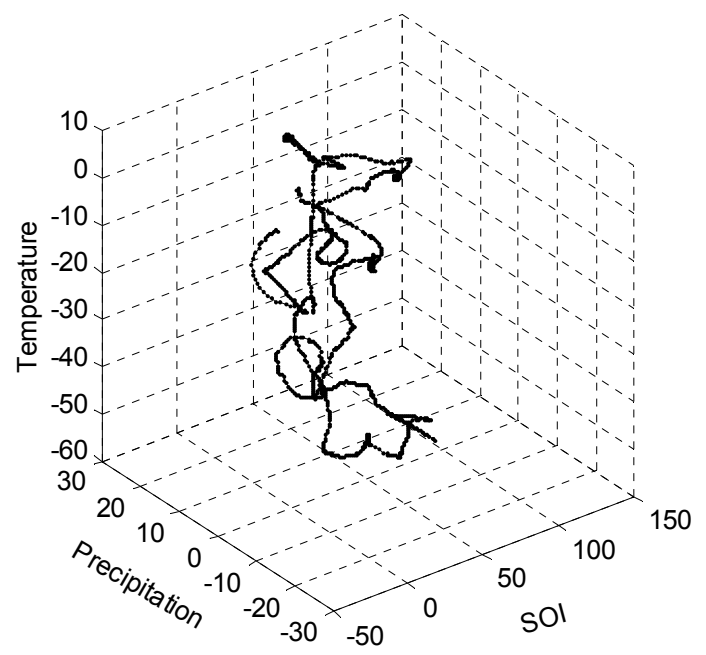

Fig. 8. Joint three-variable phase space plot of SOI, precipitation, and temperature $(\tau=0)$.

In a similar way, phase space plots can be drawn for all three variables jointly. Figure 8 shows this for all the three investigated time series. Again, the plot shows an extreme variation and no obvious structure. This may partly be due to that time series are relatively short and consequently do not cover many quasi-periodical trajectories in the phase space. However, the figure still shows the extreme nonlinearities at hand and the complicated three-dimensional structure among the three interrelated variables.

\section{Correlation dimension}

Strange attractors are typically characterized by a fractal dimension $d$ which is smaller than the number of degrees of freedom $F, d<F$ (Grassberger and Procaccia, 1983). There are several ways to estimate the fractal dimension. One of the most popular methods is the correlation dimension introduced by Grassberger and Procaccia (1983), estimated according to the algorithm given by Grassberger (1990). This algorithm is now the most commonly used way for fractal dimension estimation and, therefore, is used for the present study.

We now describe briefly the Grassberger and Procaccia algorithm. Sufficiently close trajectories will be attracted to particular regions of the phase space known as strange attractor if there are chaotic characteristics in the time series. Therefore, the points will be partially correlated (Jeong and Rao, 1996). A measure of the spatial correlation, which is commonly used for the correlation dimension method, is the correlation integral $C(N, r, m)$ which is approximated by

$C(N, r, m)=\frac{2}{N(N-1)} \sum_{j=1}^{N} \sum_{i=j+1}^{N} \Theta\left(r-\left|X_{i}-X_{j}\right|\right)$,

where $\Theta$ is the Heaviside function defined by $\Theta(s)=0$ for $s<0$ and $\Theta(s)=1$ for $s>0$. In the limit $N \rightarrow \infty$, 
$C(N, r, m) \rightarrow C(r, m)$ (Ding et al., 1993). The double sum counts the number of pairs $(i, j)$ whose distance $\left|X_{i}-X_{j}\right|$ is less than $r$. For small values of $r$, the correlation integral $C(r, m)$ exhibits a power-law dependence on $r$.

$C(r) \sim r^{d}$,

where $d$ is the correlation dimension of the attractor. The dimension $d$ of the attractor is given by the slope of $\log C(r)$ for the slope of $\log r$ according to:

$\log C(r)=d|\log r|$.

For chaotic data, $d$ will converge to a constant value with increasing embedding dimension $m$. The constant value is an estimate of the correlation dimension which measures the local structure of the strange attractor. The dimension $d$ of the strange attractor indicates at least how many variables are necessary to describe the evolution in time.

Figures 9a, 10a, and 11a show the correlation integrals $C(r)$ on logarithmic scales as a function of distance $r$ by varying embedding dimension $\mathrm{m}$ from 2 to 10 . They were calculated for the noise-reduced time series of accumulated deviations from mean for SOI, precipitation, and temperature, respectively. Figures $9 \mathrm{~b}, 10 \mathrm{~b}$, and $11 \mathrm{~b}$, on the other hand, show the local slopes $\{d \log C(r) / d \log r\}$ for the same data. In these figures, distance $r$ is normalized by its maximum value. If the slopes converge to a constant value with increase of embedding dimension $m$, the convergent slope is considered as the correlation dimension $d$ of the time series. This, thus, indicates the number of mathematical variables needed in the equation system to simulate a similar process.

For all variables, the calculated correlation integral $C(r)$ is almost the same for any embedding dimension $m$, although the integrals for temperature shows a wider range for a small distance $r$. This means the slopes of $\log C(r)$ quickly converge into a specific value with the increase of $m$. The convergent slopes for all variables are less than two, i.e. correlation dimension $d<2$, which would indicate a chaotic series in two-dimension because $d$ indicates the necessary variables to describe the time series, as mentioned before. However, this is forbidden according to Poincare-Bendixson theorem which states that a continuous one- or two-dimensional system cannot exhibit a chaotic behaviour in a bounded region of the phase space (Hense, 1987). Therefore, nonlinearly smoothed data of the accumulated deviations for all variables cannot be considered chaotic, even though they have convergent slopes in their logarithmic plots of $C(r)$ versus $r$.

\section{Conclusions and discussion}

In the paper we outlined a methodology to investigate joint phase space characteristics of several climatic variables by using ideas from dynamical systems theory. The data used were firstly treated for trends and non-normal distributions. After linear trend removal for temperature and normalization by cubic transformation for precipitation, both time series were standardized to zero mean and standard deviation of
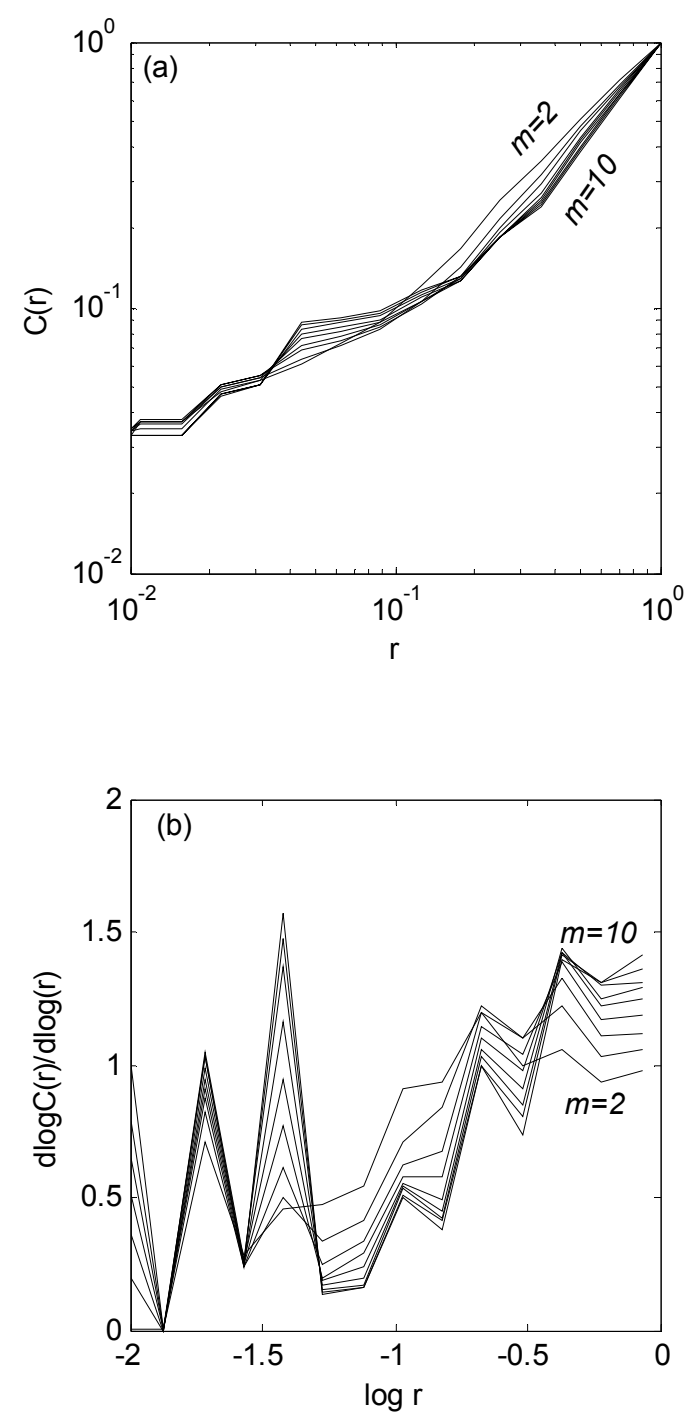

Fig. 9. (a) Correlation integral $C(r)$ versus distance $r$ for various embedding dimension $m$ of noise-reduced accumulated deviations for SOI; (b) their local slopes $d \log C(r) / d \log r$.

one. Consequently, the series are jointly homogeneous. After this the accumulated deviations from the mean were calculated for all three time series. Finally, the time series were noise reduced using an especially designed nonlinear filter.

The results from the analyses displayed rather clear phase space trajectories when treating the time series individually. However, when plotting phase space trajectories for several joint time series complicated relationships emerge. It can thus be said that the joint relationships between the three investigated variables are complex with no obvious relationships. It can thus be argued that simple data driven statistical prediction techniques may be used for individual variables (e.g. nonlinear auto-regressive techniques, local polynomials in the phase-space, etc; see e.g. Berndtsson et al., 2001). When it comes to joint co-variation of several variables, it appears that similar techniques can not be used due to the complex higher-order nonlinearities involved. The methodology 

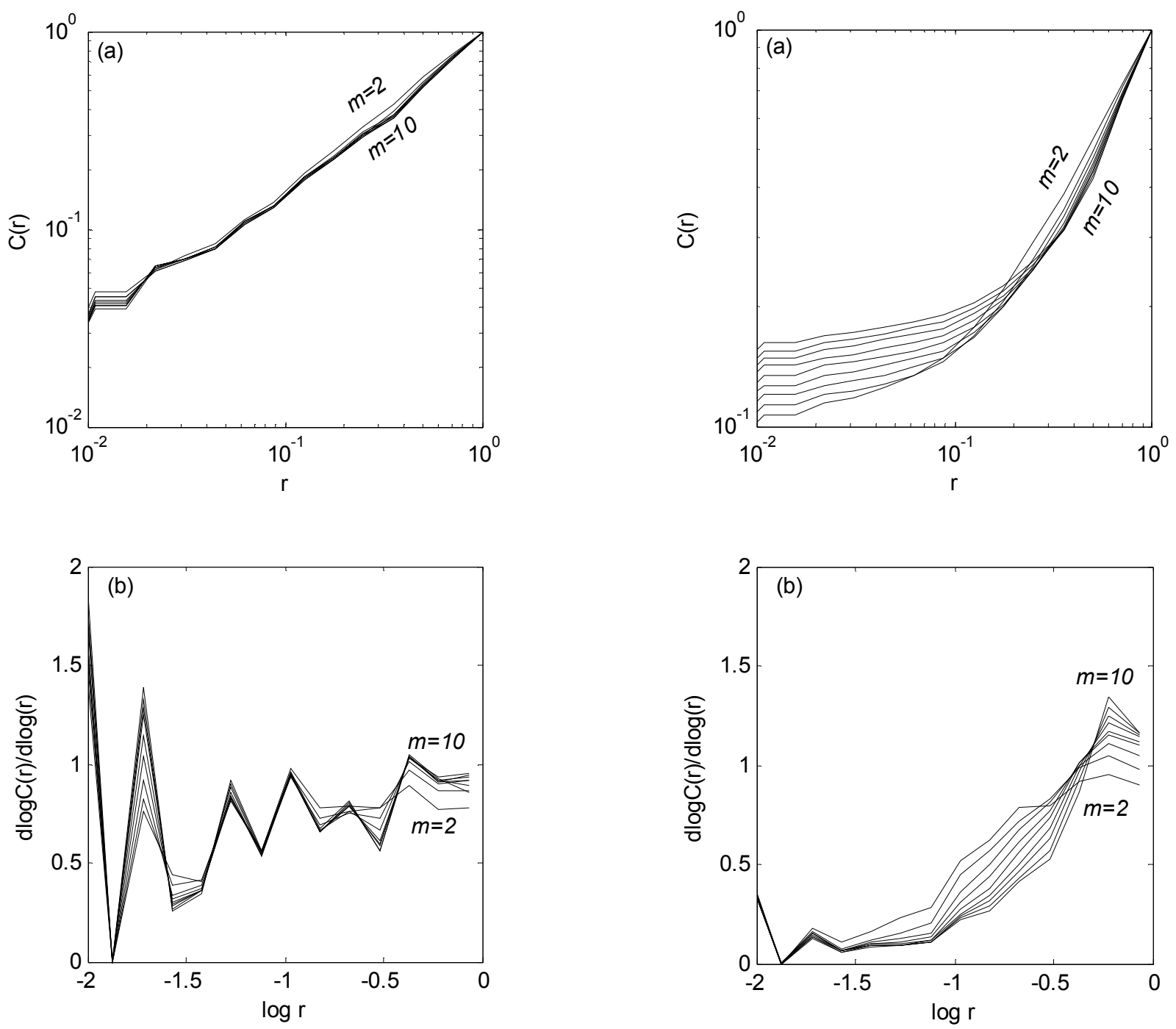

Fig. 10. (a) Correlation integral $C(r)$ versus distance $r$ for various embedding dimension $m$ of noise-reduced accumulated deviations for precipitation; (b) their local slopes $d \log C(r) / d \log r$.

presented herein, however, may serve as a basis for preliminary analysis of deterministic dynamics of several jointly interrelated variables. Because of the importance that SOI has as a driving ocean-atmosphere climatic force, it is important to investigate quantitative links between the SOI and observed local climatic and other geophysical variables even if they are not fully understood in physical terms. Observed nonlinear teleconnections may then be used as early indicators of near-term extreme weather or long-term effects on available water resources. Often these complex relationships need to consider relationships among several variables simultaneously. In this respect, the outlined methodology in this paper may be used as a first step towards this objective.

Acknowledgements. R. Berndtsson gratefully acknowledges financial support from the Swedish Research Council and the J. Gust. Richert Foundation for this study.

Fig. 11. (a) Correlation integral $C(r)$ versus distance $r$ for various embedding dimension $m$ of noise-reduced accumulated deviations for temperature; (b) their local slopes $d \log C(r) / d \log r$.

Edited by: B. Sivakumar

Reviewed by: $\mathrm{Z}$. $\mathrm{Xu}$ and another referee

\section{References}

Allan, R. J., Nicholls, N., Jones, P. D., and Butterworth, I. J.: A further extension of the Tahiti-Darwin SOI, early ENSO events and Darwin pressure, J. Clim., 4, 743-749, 1991.

Berndtsson, R., Jinno, K., Kawamura, A., Olsson, J., and Xu, S.: Dynamical systems theory applied to long-term temperature and precipitation time series, In: Trends in Hydrology, edited by Menon, J., Counc. Sci. Res. Integr., Trivandrum, India, 291-297, 1994.

Berndtsson, R., Uvo, C. B., Matsumoto, M., Jinno, K., Kawamura, A., Xu, S., and Olsson, J.: Solar-climatic relationship and implications for hydrology, Nord. Hydrol., 32, 65-84, 2001. 
Chiew, F. H. S., Piechota, T. C., Dracup, J. C., and McMahon, T. A.: El Niño/Southern Oscillation and Australian rainfall, Streamflow and drought: links and potential for forecasting, J. Hydrol., 204, 138-149, 1998

Ding, M., Grebogi, C., Ott, E., Sauer, T., and Yorke, J. A.: Plateau onset for correlation dimension: when does it occur?, Phys. Rev. Lett., 70(25), 3872-3875, 1993.

Elsner, J. B. and Tsonis, A. A.: Nonlinear prediction, chaos, and noise, Bull. Am. Meteorol. Soc., 73, 49-60, 1992.

Gordon, N. D.: The Southern Oscillation and New Zealand weather, Mon. Wea. Rev., 114, 371-387, 1986.

Grassberger, P. and Procaccia, I.: Measuring the strangeness of strange attractors, Physica, 9D, 189-208, 1983.

Grassberger, P.: An optimized box-assisted algorithm for fractal dimensions, Phys. Lett. A, 148(1-2), 63-68, 1990.

Grassberger, P., Schreiber, T., and Schaffrath, C.: Nonlinear time sequence analysis, Int. J. Bifurc. Chaos, 1, 521-547, 1991.

Hense, A.: On the possible existence of a strange attractor for the Southern Oscillation, Beit. Phys. Atmosph., 60(1), 34-47, 1987.

Jeong, G. D. and Rao, A. R.: Chaos characteristics of tree ring series, J. Hydrol., 182, 239-257, 1996.

Jin, Y.-H.: Basic study on statistical relationship between Southern Oscillation Index and precipitation in South Korea and Fukuoka, and its prediction by artificial neural network, Ph. D. Dissertation, Kyushu University, Japan, 2004.

Jin, Y.-H., Kawamura, A., and Jinno, K.: Comparison of correlation between categorized SOI and monthly precipitation at Pusan in Korea and at Fukuoka in Japan, Proc. Korea Water Resources Association, 1251-1256, 2002a.

Jin, Y.-H., Kawamura, A., and Jinno, K.: Comparison of monthly precipitation at Pusan, Mokpo, and Inchon in Korea and at Fukuoka in Japan, Proc. International Symposium on Lowland Technology, 311-316, 2002b.

Jin, F.-F, J. D. Neelin, and M. Ghil: El Niño on the devil's staircase: annual subharmonic steps to chaos, Science, 264, 70-72, 1994.

Jinno, K., Xu, S., Berndtsson, R., Kawamura, A., and Matsumoto, M.: Prediction of sunspots using reconstructed chaotic system equations, J. Geophys. Res., 100, 14773-14 781, 1995.

Kawamura, A., McKerchar, A. I., Spigel, R. H., and Jinno, K.: Chaotic characteristics of the Southern Oscillation Index time series, J. Hydrol., 204, 168-181, 1998.
Kawamura, A., Jinno, K., and Eguchi, S.: Cross-correlation between Southern Oscillation index and precipitation/-temperature in Fukuoka, Japan, Proc. of Fresh Perspectives on Hydrol. and Water Resour. in Southeast Asia and the Pacific, Christchurch, New Zealand, 32-39, 2000.

Kawamura, A., Eguchi, S., and Jinno, K.: Statistical characteristics of Southern Oscillation Index and its barometric pressure data, Annual J. Hydraulics Engineering, (in Japanese with English abstract), JSCE, 45, 169-174, 2001.

McBride, J. L. and Nicholls, N.: Seasonal relationships between Australian rainfall and the Southern Oscillation, Mon. Weather Rev., 111, 1998-2004, 1983.

Moss, M. E., Pearson, C. P., and McKerchar, A. I.: The Southern Oscillation index as a predictor of the probability of low streamflows in New Zealand, Water Resour. Res., 30, 2717-2723, 1994.

Opoku-Ankomah, Y. and Cordery, I.: Temporal variation between New South Wales rainfall and the Southern Oscillation, Int. J. Climatol., 13, 51-64, 1993.

Ropelewski, C. F. and Jones, P. D.: An extension of the TahitiDarwin southern oscillation index, Mon. Weather Rev., 115, 2161-2165, 1987.

Salas, J. D.: Analysis and modeling of hydrologic time series. In: Handbook of Hydrology, edited by Maidment, I., McGraw-Hill, New York, 20, Chap. 19, 1993.

Schreiber, T.: Extremely simple nonlinear noise-reduction method, Phys. Rev. E., 47(4), 2401-2404, 1993.

Sivakumar, B.: Chaos theory in geophysics: past, present and future, chaos, Solitons and Fractals, 19, 441-462, 2004.

Troup, A. J.: The "southern oscillation", Quarterly J. the Royal Meteo. Soc., 91(390), 490-506, 1965.

Tziperman, E., Stone, L., Cane, M. A., and Jarosh, H.: El Niño chaos: overlapping of resonances between the seasonal cycle and the Pacific ocean-atmosphere oscillator, Science, 264, 7274, 1994.

Uvo, C. B. and Graham, N.: Seasonal Runoff Forecast for Northern South America: a statistical model, Water Resour. Res., 34, 12, 3515-3524, 1998.

Uvo, C. R. B., Repelli, C. A., Zebiak, S. E., and Kushnir, Y.: The Influence of Tropical Pacific and Atlantic SST on Northeast Brazil Monthly Precipitation, J. Climate, 11, 551-562, 1998. 